\title{
Multidetector CT-generated virtual bronchoscopy: an illustrated review of the potential clinical indications
}

\author{
W. De Wever, V. Vandecaveye, S. Lanciotti, J.A. Verschakelen
}

\begin{abstract}
Multidetector CT-generated virtual bronchoscopy: an illustrated review of the potential clinical indications. W. De Wever, V. Vandecaveye, S. Lanciotti, J.A. Verschakelen. (C) ERS Journals Ltd 2004.

ABSTRACT: Multidetector computed tomography-generated virtual bronchoscopy (VB) is a recent technical development that allows visualisation of the lumen and wall of the trachea and proximal part of the bronchial tree. A dynamic image is produced that resembles what is seen with fibreoptic bronchoscopy $(\mathrm{FB})$.

Although the technique has not yet reached daily clinical practice and it can never replace $\mathrm{FB}$, performing $\mathrm{VB}$ can be useful in well-defined clinical situations.

In this paper, the value and limitations of virtual bronchoscopy will be reviewed, to illustrate the potential role of virtual bronchoscopy in the evaluation of trachea and bronchial tree pathology.
\end{abstract}

Eur Respir J 2004; 23: 776-782.
Dept of Radiology, University Hospitals Gasthuisberg, Leuven, Belgium.

Correspondence: W. De Wever, Dept of Radiology, University Hospitals Gasthuisberg, Herestraat 49, B-3000 Leuven, Belgium. Fax: 3216343765

E-mail: walter.dewever@uz.kuleuven.ac.be

Keywords: Airway multi-row computed tomography virtual bronchoscopy

Received: September 22003

Accepted after revision: January 272004
Virtual bronchoscopy (VB) is a novel computed tomography (CT)-based imaging technique that allows a noninvasive intraluminal evaluation of the tracheobronchial tree. Several studies have shown that VB can accurately show the lumen and the diameter of the trachea, the left and right main stem bronchi, and the bronchial tree down to the fourth order of bronchial orifices and branches $[1,2]$. The morphology of the carinas can be evaluated accurately and the images look very similar to that seen with fibreoptic bronchoscopy (FB).

Although VB is a promising imaging tool, this technique is not currently used in daily clinical practice and more randomised clinical trials are necessary to prove its clinical use. Nevertheless, it seems valuable to review its potential clinical indications. The purpose of this paper is to discuss and illustrate these indications based on VBs generated from the CT scans of a randomly selected group of patients.

\section{Materials and methods}

Cases were selected retrospectively and randomly from patients undergoing a multidetector row CT (MDCT) examination of the chest for various reasons. Selection was based on the presence of tracheal and bronchial abnormalities identified on the regular axial images and, when made, on additional coronal and sagittal reconstructions. MDCT was performed on a Phillips 16 slice CT (Philips, Best, the Netherlands). Technical parameters were: Kvp: 120; mAs: 150; collimation: $16 * 0.75$; pitch: 0.9 ; rotation time: 0.42 ; slice thickness: $1 \mathrm{~mm}$ and an increment of $1 \mathrm{~mm}$. MDCT was either performed with or without IV contrast administration depending on the clinical question. A proprietary virtual endoscopic software program (Endo3D; Philips) was used to reconstruct the CT data into VB images. This software program uses a volume rendering technique. A threshold value between -400 and $-600 \mathrm{HU}$ was chosen to evaluate the central bronchial tree. The more distal bronchial tree was evaluated using a threshold of $-750 \mathrm{HU}$.

Endo3D generates a perspective three-dimensional view from the inside of an anatomical structure, such as the inner wall of the colon, trachea or abdominal aorta. This insideview is generated step by step, creating a film at the end-point The thickness of each step is $2 \mathrm{~mm}$. The images are orientated with the posterior side at $12 \mathrm{o}^{\prime}$ clock, the left side at $3 \mathrm{o}^{\prime}$ clock, the anterior side at $6 \mathrm{o}^{\prime}$ clock and the right side at $9 \mathrm{o}^{\prime}$ clock.

The VB films of each patient were studied together with the regular axial CT images and when possible reconstructions in other imaging planes were also performed. VB was also compared with the report of the FB.

Volume rendering and surface rendering are the two techniques that are generally used to interpret volume. Surface rendering needs a surface description, which is extracted from the volume data. Volume rendering directly renders the volume data, without the need for such an intermediate description. Virtual bronchoscopic images are created when a threedimensional reconstruction, based on these rendering techniques, is strongly enlarged followed by a perspective computation. A computer interpolation is necessary to increase the optical resolution in order to maintain a surface whose appearance is as smooth and as natural as possible. The principal parameter influencing the quality of the reconstruction remains the use of a thin slice thickness [3]. A perspective distortion is necessary in order to choose a projection plane representing the frontal opening of the lens in the true bronchoscopy. This perspective distortion results in a more natural appearance of the objects, but is not so good for imaging in general. Defining the diameter can be difficult in the VB [3].

An important parameter for the reconstructions based on the surface or volume rendering technique is the selection of an appropriate threshold above which a surface should be presented as opaque to represent the mucosa. For surface 
rendering, a specific attenuation coefficient is used to define the air-lumen interface. Depending on the selected attenuation coefficient, surface rendering assigns all structures into air or wall. There is no transition zone. The optimal single attenuation coefficient varies between patients and pathological findings. Surface rendering is sensitive to artefact and noise [4]. With volume rendering, the entire spiral CT data set is classified. All pixels are grouped into categories on the base of their attenuation values. These groups are reconstructed as separate structures (air, wall and transition zone). With the existence of this transition zone as a separate structure, volume rendering enhances mucosal detail and adds depth and three-dimensional relief to the images [4].

A single threshold value cannot be used for the imaging of all segments of the tracheobronchial tree. While the value of $-520 \mathrm{HU}$ is appropriate for the central bronchial tree, values to $-720 \mathrm{HU}$ may be needed for the evaluation of the more distal bronchial tree. An incorrect threshold can produce artefacts like artificial endoluminal masses or openings in the mucosa [5].

The author's experience in reading VB is based on $>400 \mathrm{VB}$ performed during the last 6 months and mostly carried out in an experimental set-up, although the CT scans that were the base of the VB for the current study were done in a clinical setting. Recently, the technique was introduced into daily clinical practice and is now used in selected cases, e.g. in the evaluation of the bronchial suture in lung transplant recipients and in patients who cannot undergo FB.

The time necessary to examine the central bronchial tree with $\mathrm{VB}$ is $\sim 15 \mathrm{~min}$. There is no additional cost for the patient, since virtual bronchoscopic imaging is performed using the data from the diagnostic CT.

\section{Evaluation of bronchial stenosis}

Hoppe et al. [6] have previously examined whether coronal and sagittal reformations and VB could increase the accuracy of axial CT images to reveal tracheal and bronchial stenosis. These authors found that axial scans had an accuracy of $96 \%$, while accuracy for coronal and sagittal reconstructions was 96 and $96.5 \%$, respectively. When VB was performed accuracy increased to $98 \%$ (fig. 1). They also showed that there was a good correlation between VB and FB when the grade of tracheobronchial stenosis resulting from either endoluminal pathology or external impression was estimated. These correlations were also better than when tracheobronchial stenosis was graded on axial slices or on reformatted images [6]. This was confirmed by BURKE et al. [7] who showed that VB was accurate in assessing width and length of fixed airway lesions. Correlations between FB and VB were excellent when shape and contour of the stenosis were studied, while stenosisto-lumen ratios were found to be within $10 \% \quad(\mathrm{SD}=8)$. However, when based on dynamic airway lesions, such as immobile true vocal cords, innominate artery compression and laryngotracheomalacy, VB was unable to detect the cause of airway obstruction [7].

\section{Bronchogenic carcinoma}

CT is routinely performed to detect, stage and follow-up primary lung tumours. In most cases only axial CT slices are used for evaluation although with the introduction of newer and faster computers additional two-dimensional and threedimensional reconstructions are often made to improve the diagnosis, description and staging of these tumours. FINKELSTEIN et al. [8] examined the potential role of VB and
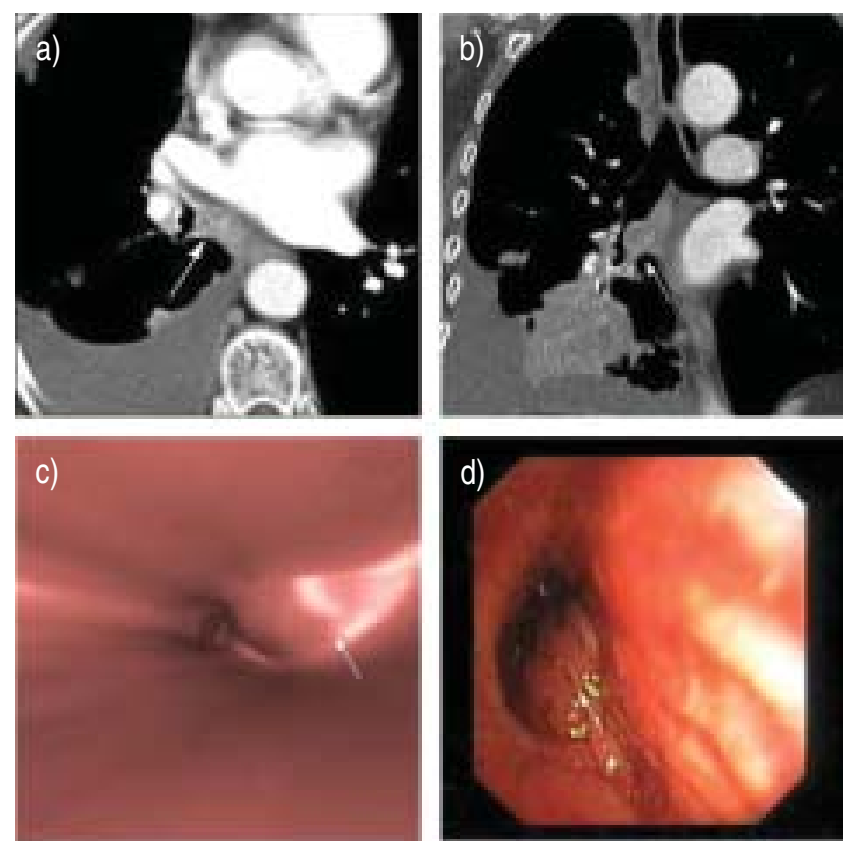

Fig. 1.-A chest computed tomography (CT) was performed on an 81-yr-old with dyspnoea and a right-sided pleural effusion, to exclude a tumoral process. a) Axial CT image, b) coronal reconstruction, c) virtual bronchoscopic (VB) and d) a fibreoptical bronchoscopic (FB) view of the right intermediate bronchus (RIB). The VB shows narrowing of the RIB due to a mass (arrow), which is extending into the lumen at the left side of this bronchus. This mass is suspicious for a bronchial tumour. The finding is confirmed during FB, and good correlations were found between both techniques, in both the evaluation of the bronchial diameter and in the evaluation of the tumour.

found that the sensitivity of VB was $100 \%$ for detection of obstructive lesions (figs 2 and 3), and 83\% for endoluminal nonobstructive lesions, but the sensitivity for mucosal abnormalities was $0 \%$. The specificity of VB was $100 \%$.

In most cases, VB is able to depict direct tumour signs, such as a tumoral mass, a wall irregularity or a loss of cartilages. Indirect signs, such as stenosis or obstruction, compression or swelling, can often be visualised. However, mucosal infiltration, vascular dilatation and necrosis are usually missed [3].

RAPP-BERNHARDT et al. [9] identified endoluminal tumours by VB and grade of the stenosis was calculated and compared with measurements obtained from FB, axial CT slices, multiple plane reconstruction and minimal intension projection reconstructions. No significant differences were found when VB and FB were compared. However, axial CT slices, multi planar reconstructions and minimal intensity projections showed poorer results with over- or underestimation of the stenoses [9].

These preliminary evaluations indicate that VB may be a promising and noninvasive modality for identifying and grading bronchial obstructions and endoluminal lesions. However, VB does not currently enable detection of subtle mucosal lesions and, for this reason, this modality is not appropriate for identifying premalignant lesions in the respiratory tract [8].

In addition, very viscous secretions in the bronchial tree or coagulated blood can mimic a stenosis or occlusion, and only a careful comparison with the axial CT images makes differentiation possible [1]. However, excessive smoothing of the airways generated by the computer algorithm can produce false-negatives [10]. An important advantage of VB over FB is that axial and, when performed, reformatted images are available. Combining the information from these images allows not only the intraluminal proliferation of the tumour to be appreciated, but also the extraluminal extension of the 

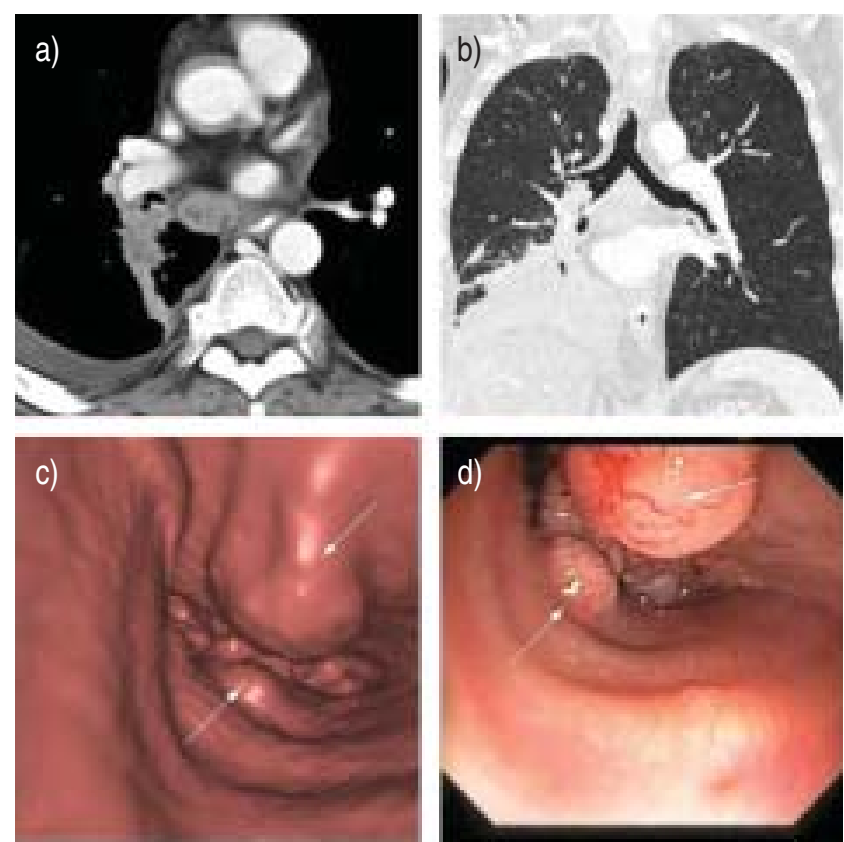

Fig. 2. - A 52-yr-old patient with a tumour of the tongue and a suspicious lesion in the right lung (an invasive spinocellular carcinoma). An axial image (a) and a coronal reconstruction (b) of the right hilum are shown, confirming the presence of the mass, which is invading the tracheobronchial tree at the level of the secondary carina. Virtual bronchoscopy (VB) (c) shows a polypoid tumoral proliferation (arrow) at the distal part of the right main stem bronchus and secondary carina. VB could pass along this tumoral process into the right intermediate bronchus. Fibreoptic bronchoscopy (d) shows the same lesion (arrow), but could not pass the lesion.

mass and its relation to the bronchial tree. Another advantage of $\mathrm{VB}$ is that the bronchial tree, distal to an obstruction that cannot be passed with FB, can be evaluated [7, 11-13]. In this way the length of the stenosis can be estimated, which is of course impossible when the fibreoptic bronchoscope cannot pass the stenosis. VB can also look at the distal border of an
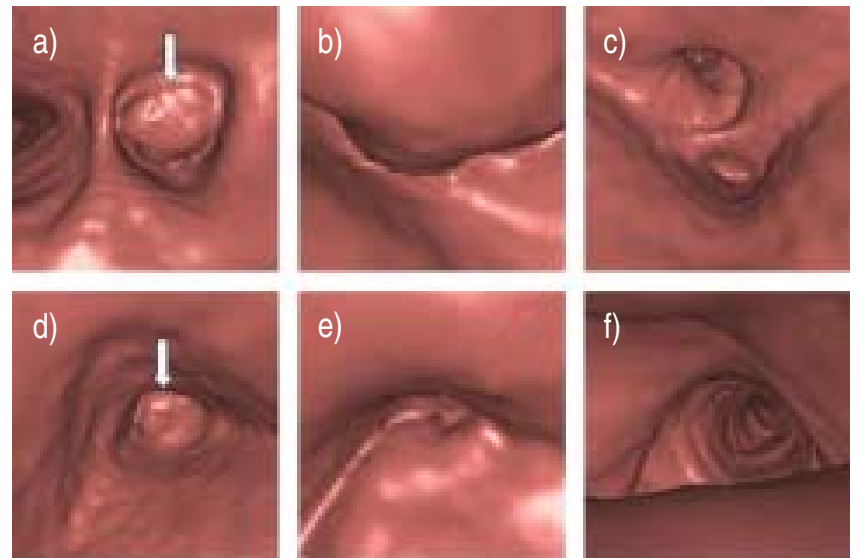

Fig. 3. - A 62-yr-old patient with left-sided lobectomia superior and tumour recurrence at the left main bronchus. Virtual bronchoscopy (VB) of the left main bronchus shows the endoluminal proliferation of the tumour on the dorsal side of the left main bronchus (a; arrow), which is occluding almost the entire lumen. The VB passes along the tumour (b) and the secondary carina (c). By retroflexing the virtual bronchoscope the distal aspect of the endoluminal tumour (d; arrow) is seen on the dorsal side of the left main bronchus. The VB view, when passing the tumour from distal to proximal through a very small passage (e) and a retrograde view into the trachea (f) are also shown. endoluminal mass or stenosis by "retroflexing the virtual bronchoscope" [7, 11-13]. In contrast to FB, VB is able to visualise the bronchial tree down to the fourth or fifth generation of bronchi. Finally, VB can provide a road map for $\mathrm{FB}$ and guide transbronchial biopsy and endobronchial treatment [1].

\section{External compression}

VB can be useful to visualise external compressions on the bronchial wall not involving the mucosa (fig. 4). These compressions can be caused by normal anatomical structures (i.e. aortic arch, oesophagus) or can be caused by pathological structures (i.e. extraluminal tumour, enlarged lymph nodes, fibrotic masses). Correlating the position of the virtual bronchoscope with the axial CT images usually allows the exact localisation and cause of the external compression to be defined. However, small compressions are difficult to detect and may be underestimated in $25 \%$ of patients [12]

\section{Anatomical malformation and variants}

VB may also be used in selected cases to study congenital or acquired anatomical malformations, e.g. tracheal or bronchial diverticula (fig. 5), and tracheal bronchi. These are often incidental findings and treatment is not necessary in most cases. When a detailed study of the malformation is required VB can be performed and can replace the more invasive FB. If the malformation was depicted on a $\mathrm{CT}$ examination, no additional radiation to the patient is necessary, since the VB images can be calculated from the existing CT data.
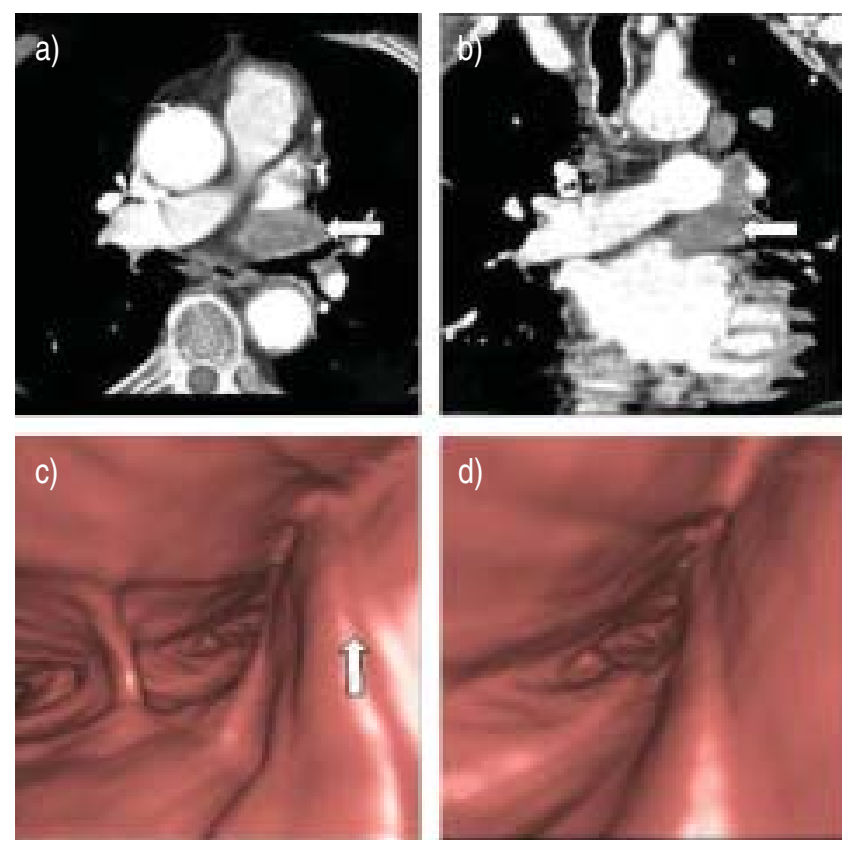

Fig. 4. - A 75-yr-old patient with a tumoral process in the left hilum and with hilar adenopathies. a) Axial computed tomography images and b) coronal reconstructions show the enlarged lymph nodes in the left hilum (arrow), especially anterior to the left main bronchus. Virtual bronchoscopy (c, d) shows a narrowing of the left main bronchus due to an impression on the ventral part of this left main bronchus (arrow). This impression is caused by the enlarged hilar lymph nodes. 

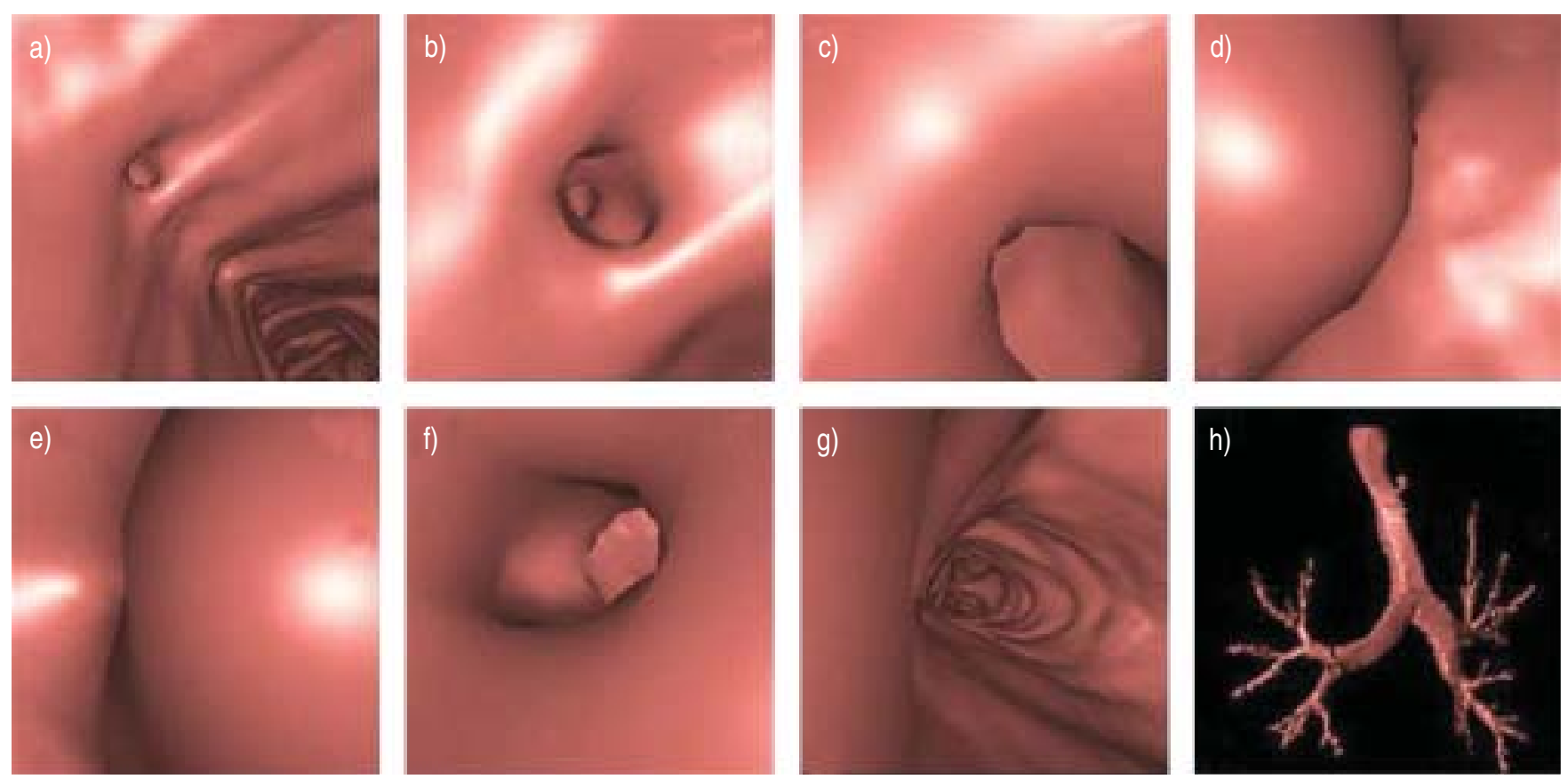

Fig. 5. - A 59-yr-old patient with left-sided malignant mesothelioma and a trachea diverticle as an incidental finding. Virtual bronchoscopy shows a small opening on the dorsal side of the trachea $(a, b)$, the VB entering the opening (c, d), retroflexing the virtual bronchoscope in the diverticle $(\mathrm{e}, \mathrm{f})$ and re-entering of the VB into the trachea $(\mathrm{g})$. Three-dimensional reformatting (h) shows a small air-containing lesion protruding from the tracheal wall, the trachea diverticle.

\section{Evaluation of trachea and bronchi in children}

Indications for flexible bronchoscopy are, especially in very young children, restricted, because of its invasive character and also owing to the inevitable general anaesthesia. Besides the radiation that usually can be kept to a minimum in children, a VB is less invasive than a FB because a simple sedation is usually sufficient to perform the CT scan. Nevertheless, indications for a VB in children should be defined clearly and individually. The image quality of a VB in neonates is indeed mostly inferior to that in adults because of the smaller diameter of the airways [14]. In addition, artefacts due to respiratory motion or mucus plugs can mimic pathology. Respiratory motion is often a problem and is related to the fact that it is usually impossible to perform the examination during one breath hold. Fortunately, new multidetector CT techniques allow very short acquisition times and, in addition, reduce radiation exposure [15]. A potential indication for a VB in children is to rule out bronchial abnormalities, such as stenosis (figs 6 and 7) or aberrant bronchi, as a cause of recurrent infiltrates seen on a chest radiograph. KIRCHNER et al. [16] showed that VB could avoid additional diagnostic bronchoscopy in a high percentage $(73 \%)$ of cases.

\section{Guidance of transbronchial biopsy}

Blind lymph nodal transbronchial biopsies by means of FB do not have high sensitivity. The success rate is $94 \%$ for lesions visible during the procedure [17], but only $50 \%$ for lymph nodes and tumours that are not visible [15]. When the mucosa appears normal, the bioptical sampling can only be approximated. This is the reason false-positives biopsies are very rare, but false-negatives are numerous [17]. This delay in staging induces a delay in the start of the chemotherapy, because more invasive staging procedures are necessary [18]. Information from VB in combination with axial CT images and coronal or sagittal reconstructions can be helpful in determining the best location for a transbronchial biopsy, especially for lesions that do not affect the mucosa nor distort the airways [18, 19]. VB can also help the bronchoscopist define the optimum pathway for passing instruments into lesions beyond the field of view [12, 20,21]. It has been shown that VB significantly increases the success rate of transbronchial biopsy in pretracheal and hilar enlarged lymph nodes and in small $(<1.5 \mathrm{~cm})$ lymph nodes [18]. However, for subcarinal and aortopulmonary window lymph nodes sampling, this increase was not significant [18]. New software programs making the airway wall semitransparent, revealing extrabronchial structures with a perspective view, are promising and can help to identify the lymph nodes adjacent to the trachea and bronchial tree. Research work is also being performed on software for assisted needle aspiration where VB is no longer a passive guide but actively guides the transbronchial biopsy.

\section{Pre- and postoperative assessment}

\section{Preoperative planning}

VB can be helpful to guide endobronchial procedures, such as laser photocoagulation, brachytherapy, endobronchial cryotherapy and placement of endobronchial stents [11, 19].

VB can provide exact information on luminal diameter and length of stenosis, which is necessary for accurate stent placement (fig. 8) [7, 22]. VB and three-dimensional images also provide an easier global understanding of the tracheobronchial status [23] and the relationship with the surrounding normal or pathological structures.

\section{Postoperative evaluation}

VB is not only helpful during preoperative staging, but can be used also as a follow-up imaging tool in the postoperative 

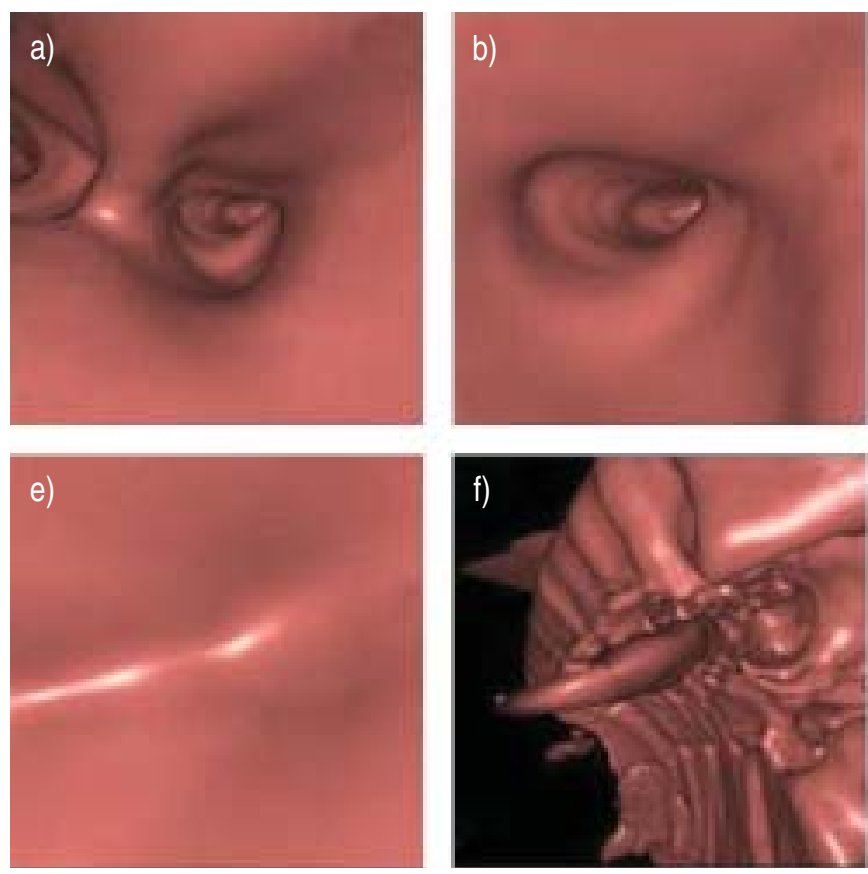
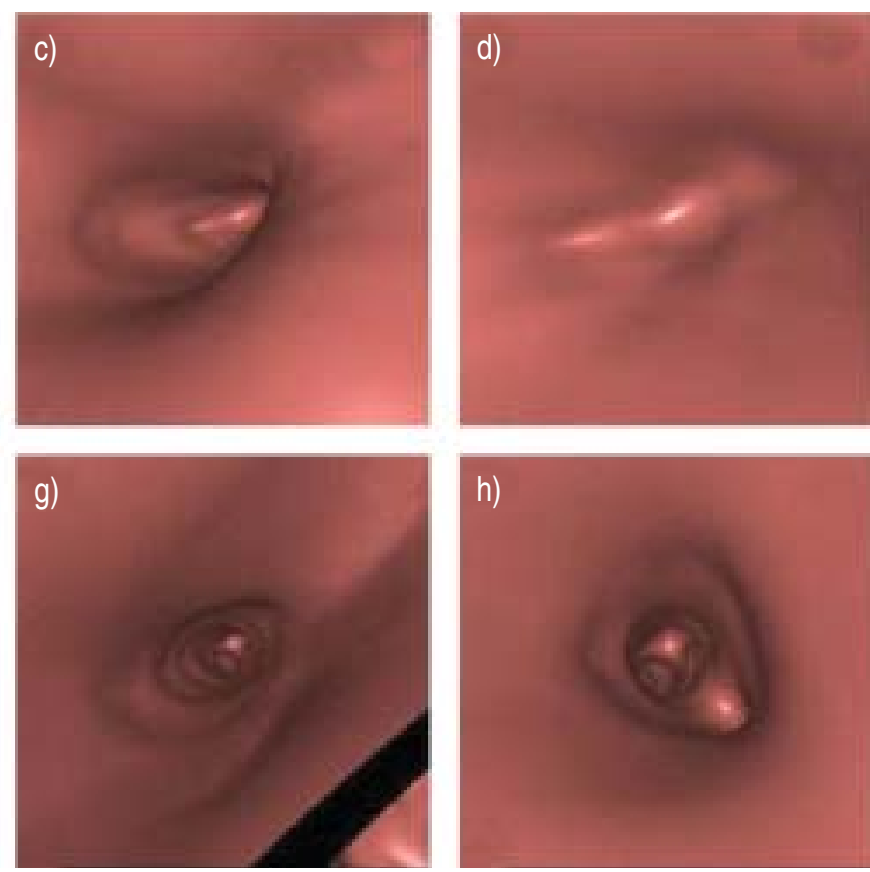

Fig. 6. - A 2-yr-old child with congenital narrowing of the left main bronchus and retro-obstructive emphysema of the left lung. Virtual bronchoscopy shows a normal carina (a) and an almost 100\% narrowing of left main bronchus (b-d). The narrowing was passed virtually (f, g), and distal from this narrowing the bronchial tree was open and normal (h).

assessment of patients with treatment of the bronchial tree in the evaluation of stenoses, and evaluation of the position and permeability of a stent (fig. 9) [11, 12, 24].

Evaluation of the surgical suture after lung transplantation, lobectomy and pneumectomy is even possible. MCADAMS et al. [11] showed that VB was slightly more accurate than axial CT for the diagnosis of clinically relevant stenosis at bronchial anastomosis in lung transplant recipients.
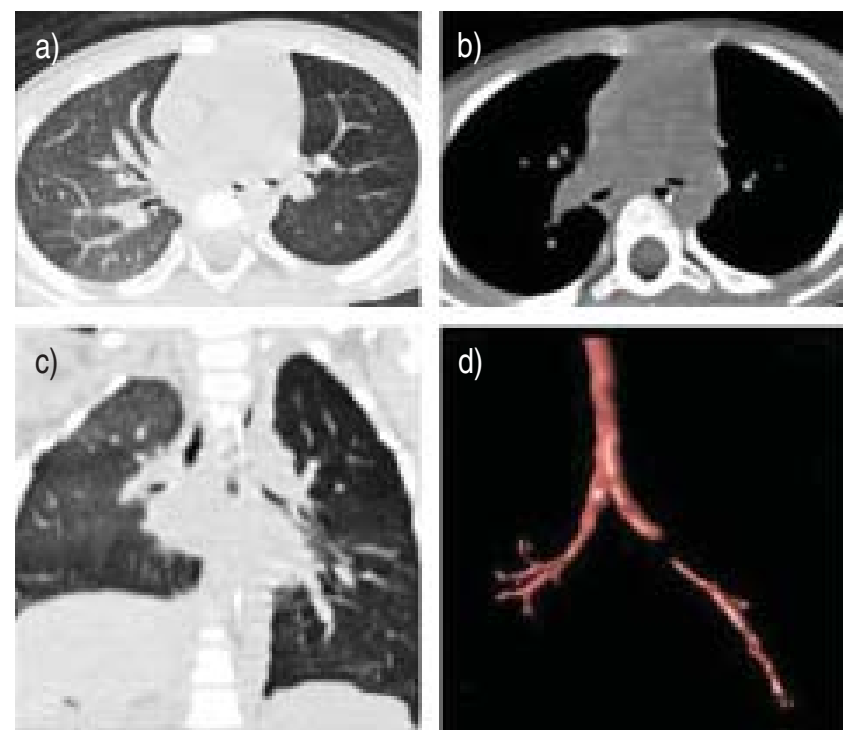

Fig. 7. - The same 2-yr-old child with congenital narrowing of the left main bronchus and retro-obstructive emphysema of the left lung as in fig. 6. Axial CT images (a, b) and coronal reconstructions (c) show the retroobstructive air trapping of the left lung. The threedimensional reformatted images (d) show the congenital stenosis of the left main bronchus.

\section{Research applications and educational tool}

Besides the clinical indications, VB may also be used in educational and research applications, to teach and learn the tracheobronchial anatomy as it appears at bronchoscopy. This "flight simulator" not only helps training pulmonologists to learn how to perform a FB but can also help them, when interpreted immediately before the fibreoptic procedure, to
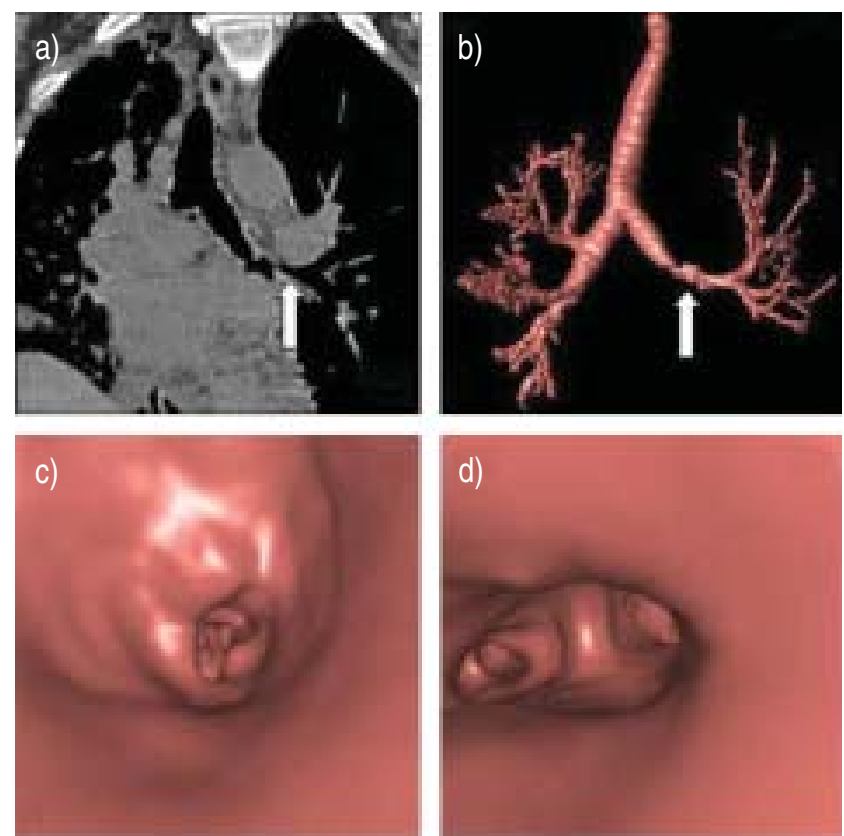

Fig. 8. - A 60-yr-old patient with left lung transplantation. Coronal reconstructions (a) and three-dimensional reformatted imaging (b) reveal the important stenosis located at the suture of the transplanted lung (arrow). Virtual bronchoscopy (c, d) shows the narrowing involving more than two thirds of the lumen and can pass the stenosis to examine the (normal) distal bronchial tree. 

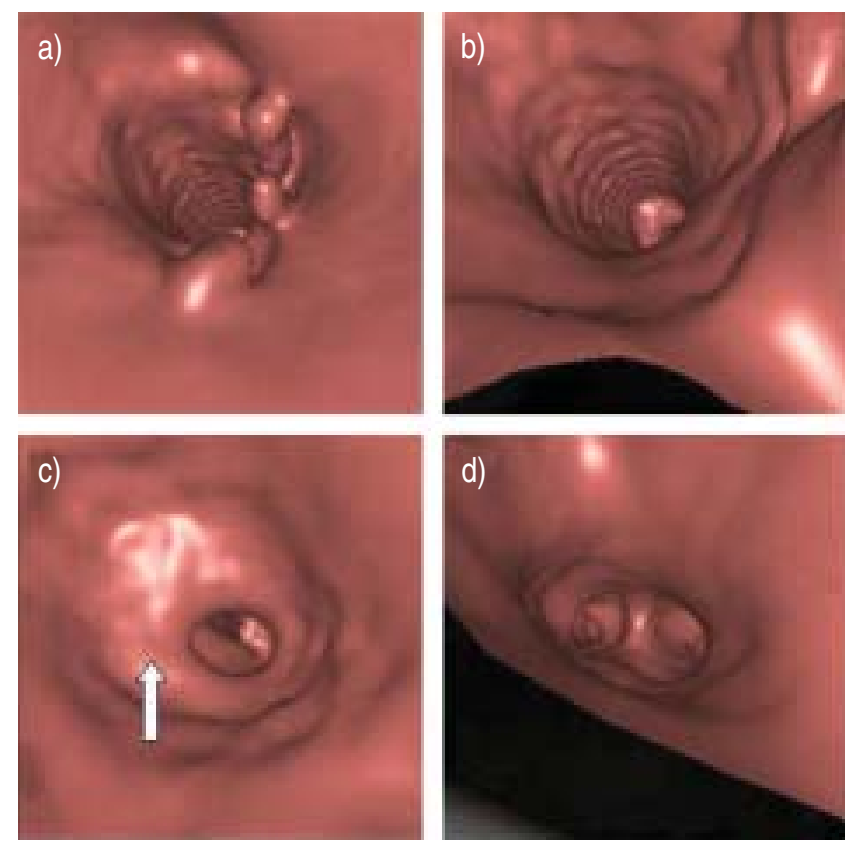

Fig. 9. - A 60-yr-old patient with left lung transplantation and a stent placed to treat a postoperative stenosis. Virtual bronchoscopy shows the stent $(\mathrm{a}, \mathrm{b})$ in the left main bronchus that became loose, proximally. The proximal part of the stent is open but there is a stenosis in the distal part due to granulomatous tissue (c; arrow). The bronchial tree distal to the stenosis is normal (d).

perform this invasive procedure faster and more efficiently [3, $25,26]$.

\section{Conclusion}

VB is a novel technique for noninvasive evaluation of the tracheobronchial tree. In comparison with real bronchoscopy, VB has the advantage of being a noninvasive procedure that can visualise areas inaccessible to the flexible bronchoscope. Preliminary evaluation indicates that VB may be a promising and noninvasive modality for identifying bronchial obstructions and endoluminal lesions, as well as for assessing the tracheobronchial tree beyond stenoses.

However, VB does not currently enable the detection of subtle mucosal lesions [2, 8]. In addition, the patient is exposed to radiation and although $\mathrm{VB}$ is accurate $[11,12,23,27]$, it is not $100 \%$. False-positives and false-negatives occur. Feasibility of virtual bronchoscopy also depends on the availability and use of the CT hardware and software, and on the observer's experience [28].

Virtual bronchoscopy will never replace actual bronchoscopy, but in selected cases it can assist and direct fibreoptical bronchoscopy and can sometimes give additional information. It can be expected that in the near future, this technique will be used on a daily basis [3]

\section{References}

1. Polverosi R, Vigo M, Baron S, Rossi G. Evaluation of tracheobronchial lesions with spiral CT: comparison between virtual endoscopy and bronchoscopy. Radiol Med (Torino) 2001; 102: 313-319.

2. Aquino SL, Vining DJ. Virtual bronchoscopy. Clin Chest Med 1999; 20: 725-730.
3. Rogalla P, Meiri N. Virtual endoscopy of the trachea and the bronchi. In: Rogalla P, Terwisscha van Scheltinga J, Hamm B, eds. Virtual endoscopy and related 3D techniques. Berlin, Springer, 2001; pp. 45-75.

4. Hopper KD, Iyriboz AT, Wise SW, Neuman JD, Mauger DT, Kasales CJ. Mucosal detail at CT virtual reality: surface versus volume rendering. Radiology 2000; 214: 517-522.

5. Maniatis P, Triantopoulou C, Tsalafoutas I, et al. Threshold selection in virtual bronchoscopy: phantom study and clinical applications. Lausanne, Scientific Exhibits, ESTI 2003.

6. Hoppe H, Walder B, Sonnenschein M, Vock P, Dinkel HP Multidetector CT virtual bronchoscopy to grade tracheobronchial stenosis. Am J Roentgenol 2002; 178: 1195-1200.

7. Burke AJ, Vining DJ, McGuirt WF Jr, Postma G, Browne JD. Evaluation of airway obstruction using virtual endoscopy. Laryngoscope 2000; 110: 23-29.

8. Finkelstein SE, Summers RM, Nguyen DM, Stewart JH 4th, Tretler JA, Schrump DS. Virtual bronchoscopy for evaluation of malignant tumors of the thorax. $J$ Thorac Cardiovasc Surg 2002; 123: 967-972.

9. Rapp-Bernhardt U, Welte T, Doehring W, Kropf S, Bernhardt TM. Diagnostic potential of virtual bronchoscopy: advantages in comparison with axial CT slices, MPR and mIP? Eur Radiol 2000; 10: 981-988.

10. Rodenwaldt J, Kopka L, Roedel R, Margas A, Grabbe E. $3 \mathrm{D}$ virtual endoscopy of the upper airway: optimization of the scan parameters in a cadaver phantom and clinical assessment. J Comput Assist Tomogr 1997; 21: 405-411.

11. McAdams HP, Palmer SM, Erasmus JJ, et al. Bronchial anastomotic complications in lung transplant recipients: virtual bronchoscopy for noninvasive assessment. Radiology 1998; 209: 689-695.

12. Fleiter T, Merkle EM, Aschoff AJ, et al. Comparison of realtime virtual and fiberoptic bronchoscopy in patients with bronchial carcinoma: opportunities and limitations. $\mathrm{Am}$ J Roentgenol 1997; 169: 1591-1595.

13. Liewald F, Lang G, Fleiter T, Sokiranski R, Halter G, Orend KH. Comparison of virtual and fiberoptic bronchoscopy. Thorac Cardiovasc Surg 1998; 46: 361-364.

14. Konen E, Katz M, Rozenman J, Ben-Shlush A, Itzchak Y, Szeinberg A. Virtual bronchoscopy in children: early clinical experience. Am J Roentgenol 1998; 171: 1699-1702.

15. Lam WK, So SY, Hsu C, Yu DY. Fibreoptic bronchoscopy in the diagnosis of bronchial cancer: comparison of washings, brushings and biopsies in central and peripheral tumours. Clin Oncol 1983; 9: 35-42.

16. Kirchner J, Laufer U, Jendreck M, Kickuth R, Schilling EM, Liermann D. Virtual bronchoscopy in the child using multislice CT: initial clinical experiences. Rontgenpraxis 2000; 53 : 87-91.

17. Martini N, McCormack PM. Assessment of endoscopically visible carcinomas. Chest 1978; 73: 718-720.

18. Hopper KD, Lucas TA, Gleeson K, et al. Transbronchial biopsy with virtual CT bronchoscopy and nodal highlighting. Radiology 2001; 221: 531-536.

19. Bricault I, Ferretti G, Cinquin P. Registration of real and CT-derived virtual bronchoscopic images to assess transbronchial biopsy. IEEE Trans Med Imaging 1998; 17: 703714.

20. Haponik EF, Aquino S, Vining DJ. Virtual bronchoscopy. Clin Chest Med 1999; 20: 201-217.

21. McAdams HP, Goodman PC, Kussin P. Virtual bronchoscopy for directing transbronchial needle aspiration of hilar and mediastinal lymph nodes: a pilot study. Am J Roentgenol 1998; 170: 1361-1364.

22. Cicero R, Criales JL, Lorenzo JM. Flexible bronchoscopy and helical computer tomography with three-dimensional reconstructions in a non-small cell carcinoma of the lung. J Bronchol 1998; 5: 98-103.

23. Gaeta M, Pandolfo I, Volta S, et al. Bronchus sign on CT in peripheral carcinoma of the lung: value in predicting results 
of transbronchial biopsy. Am J Roentgenol 1991; 157: 11811185.

24. Ferretti GR, Kocier M, Calaque O, et al. Follow-up after stent insertion in the tracheobronchial tree: role of helical computed tomography in comparison with fiberoptic bronchoscopy. Eur J Radiol 2003; 13: 1172-1178.

25. Vining DJ, Liu K, Choplin RH, Haponik EF. Virtual bronchoscopy: relationships of virtual reality endobronchial simulations to actual bronchoscopic findings. Chest 1996; 109: 549-553.

26. Buthiau D, Antoine E, Piette JC, Nizri D, Baldeyrou P,
Khayat D. Virtual tracheo-bronchial endoscopy: educational and diagnostic value. Surg Radiol Anat 1996; 18: 125-131.

27. Ferretti GR, Knoplioch J, Bricault I, Brambilla C, Coulomb M. Central airway stenoses: preliminary results of spiral-CT-generated virtual bronchoscopy simulations in 29 patients. Eur Radiol 1997; 7: 854-859.

28. Neumann K, Winterer J, Kimmig $\mathbf{M}$, et al. Real-time interactive virtual endoscopy of the tracheo-bronchial system: influence of CT imaging protocols and observer ability. Eur J Radiol 2000; 33: 50-54. 